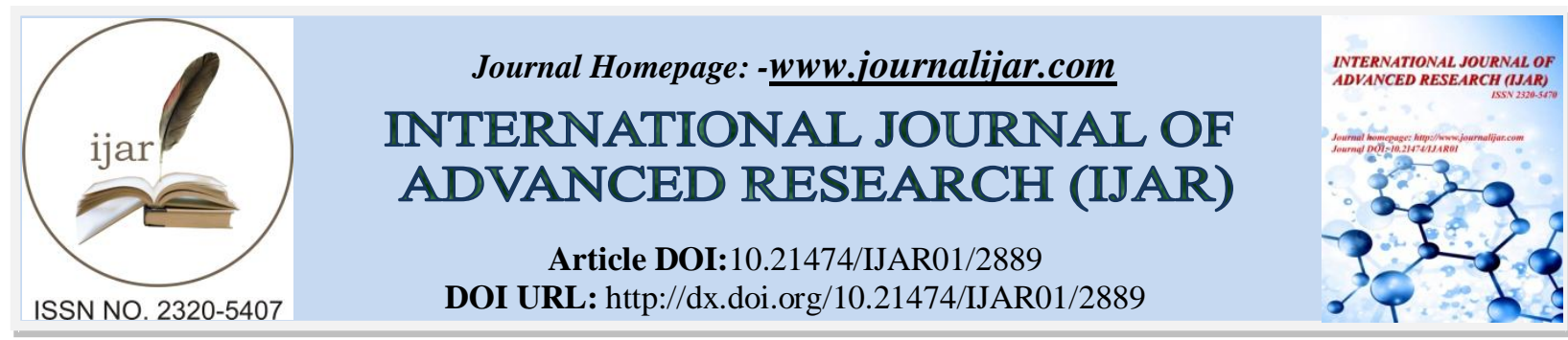

RESEARCH ARTICLE

\title{
BILATERAL SEQUENTIAL LUNG TRANSPLANT FOR PULMONARY ALVEOLAR MICROLITHIASIS.
}

Dr. Ibrahim Saud Al Tamimi, Dr. Mohammed Ahmed Alsahabi and Dr. Feras Khalid Aledrees

College of Medicine, Alfaisal University, Riyadh, Saudi Arabia.

\section{Manuscript Info}

Manuscript History

Received: $x x x x x x x x x x x x x x x$

Final Accepted: $x x x x x x x x x x x$

Published: $x x x x x x x x x x x x x x x$

Key words:-

Pulmonary Alveolar Microlithiasis;

Double lung transplant

\begin{abstract}
Pulmonary alveolar microlithiasis is an uncommon infiltrative pulmonary disease characterized by deposition of microliths in the alveoli. Here, we present the case of a 45-year-old man with PAM who is a successful lung transplant recipient with end-stage PAM

Introduction: Pulmonary alveolar microlithiasis (PAM) is a rare idiopathic lung disease, characterized by the formation and accumulation of tiny, round corpuscles called "microliths" that consist primarily of calcium and phosphorus mixed with small amounts of magnesium and aluminum ${ }^{1}$. Most of the reported cases are of patients between 20 and 40 years of age. Autosomal recessive inheritance has been suggested to be a possible cause of the disease. Clinical features vary, and some patients may be asymptomatic for a long time until pulmonary function testing begins to demonstrate lung impairment along with progressive fibrosis and the development of a restrictive pulmonarydefect culminating in cardiorespiratory decompensation. ${ }^{2}$ Currently, there is no medical therapy capable of definitively changing the progression of the disease. Lung transplantation is required once end-stage lung disease is established.
\end{abstract}

Copy Right, IJAR, 2016,. All rights reserved.

\section{Case Description:-}

Mr. Ibrahim Mohammed is a 45-year-old gentleman known case of pulmonary alveolar microlithiasis, severe pulmonary hypertension and respiratory failure on home oxygen. He was admitted on 04 February 2016 because of decline in lung function and exertional dyspnea for 2 weeks, at that time a suitable lung transplant donor was available. There was no history of smoking, no family history of PAM. His crossmatch was negative for both T cell and B cell. The induction therapy was started with methylprednisolone $500 \mathrm{mg}$ for each lung and CellCept, and broad-spectrum antibiotics meropenem, colistin, and vancomycin as per lung transplant protocol. The intraoperative course remained smooth, was done on ECMO without any major intraoperative issue. The ischemia time for the right lung was 6 hours and for the left lung was 6.5 hours.

Posttransplant, there was a mild reperfusion injury and was shifted to ICU intubated with minimal inotropic requirements. He was successfully weaned off from inotrope and extubated on the next day with stable vitals and good recovery. On 08 February 2015, he had tonic clonic seizures. For that he had to be re-intubated. The labwork was normal, electrolytes were normal. Neurology was taken onboard and MRI was advised. His MRI showed bilateral predominant frontoparietal area of cortical signal abnormality, diffuse sulcal FLAIR hyperintensity noted,

Corresponding Author:-Dr.Ibrahim Saud AITamimi.

Address:-College of Medicine, Alfaisal University, Riyadh, Saudi Arabia. 
which is of uncertain etiology. Since there was no clear cause for his tonic clonic seizure, his tacrolimus was switched to cyclosporine and CellCept was changed to azathioprine.

He was successfully extubated in the next 2 days and then shifted to the floor. His respiratory cultures were negative except legionella for which he was treated. The transbronchial biopsy showed no evidence of any cellular rejection. He was discharged home in a stable condition on 18 February 2016 with a followup clinic visit in 1 week with repeat lab imaging and spirometry. Physical examination revealed athin, chronically ill appearing male with respiratory rate of $20 \mathrm{bpm}$. Prominent inspiratory crackles were noted bilaterally,other examination were unremarkable. The spirometry done before discharge showed FEV1 1.92, FVC 2.26, FEV1/FVC ratio 85.07. Specimen is received in formalin labeled right lower lobe. It consists of multiple fragments of brownish and tan soft tissue, measuring in aggregate $0.4 \times 0.4 \times 0.1 \mathrm{~cm}$.

\section{Discussion:-}

Although the first cases of PAM were described by Malphighi, the disease was first named by Phur. The disease exists on all continents, without regional or ethnic differences, although most of the cases (37\%) have been reported in Europe and Asia Minor. Turkey is the country with the highest prevalence (16.3\%), followed by Italy and the USA. The etiology of the condition remains unknown, although some theories have been posited. One theory is based on an exudate that is not easily absorbed and that is formed as a result of an abnormal inflammatory response to infections or irritants, ultimately undergoing calcification. Males and female are affected with equal frequency. There appears to be a family association in almost half of the reported cases.

A striking feature of this disease is discordance between the clinical and radiographic manifestation, many patient display only minor symptoms although impressive radiographic manifestation. Those patient who are symptomatic typically complain of dyspnea and nonproductive cough, and the disease may develop slowly into corpulmonale.

The typical picture of PAM on chest X-ray is of fine, sand-like calcific micronodules (sandstorm lung) diffusely infiltrating both lungs; these characteristics are usually observed in the middle and lower zones. CT scans demonstrate micronodular calcific densities with a greater concentration in the subpleural parenchyma and along the bronchovascular bundles, whereas high resolution CT scans show thickening of the lobular septae with a distribution of microliths along the septae and around the centrilobular distal bronchioles.

Figure 1:-Chest radiograph (A) and computed tomographic scan (B) on admission.

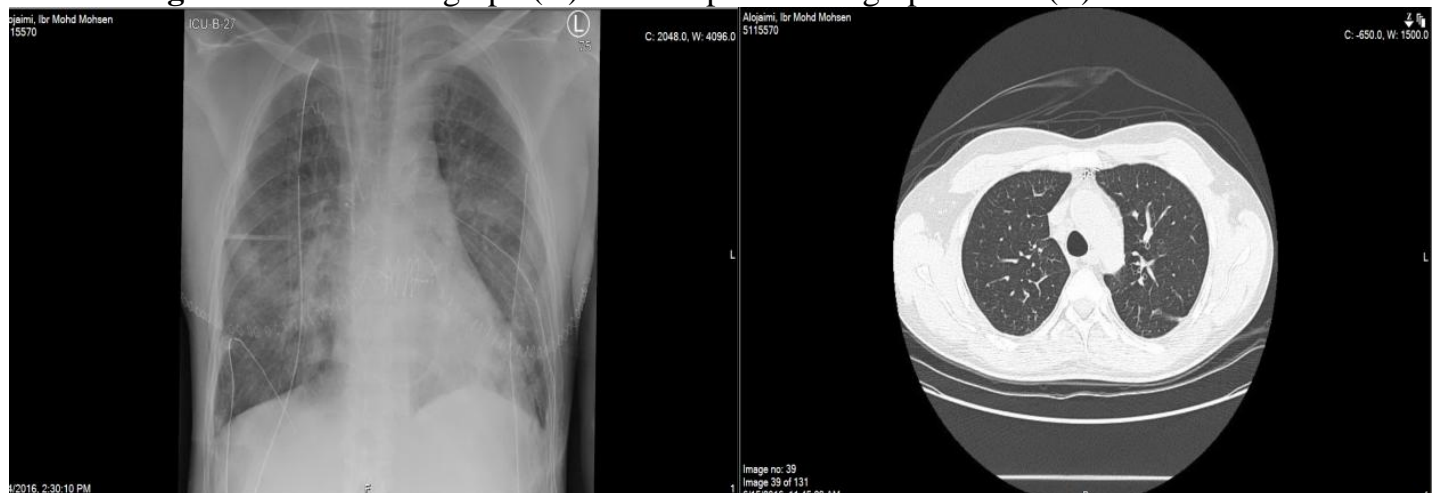

Considering lung transplantation as a treatment method in PAM cases instead of medication or artificial respiratory support treatments, both of which are known to be ineffective, seems rational. The differences observed between the preoperative and postoperative periods demonstrated the importance of performing bilateral lung transplantation in such cases regardless of the recurrence risk.

Systemic corticosteroids, calcium-chelating agents and serial bronchopulmonary lavage have been shown to be ineffective and are used as palliative treatments. In the only postoperative death reported after bilateral lung transplantation, prolonged cardiopulmonary bypass (six hours) in combination with extensive pleural involvement and increased vascularity led to uncontrolled hemorrhagic complications. Shigemura et al. have reported the oldest successful lung transplant recipient, with ten years of follow-up. 
Bilateral lung replacement is preferred to unilateral replacement, because the replacement of only one lung might result in persistent shunting of blood through the native lung, as PAM leads to filling of the alveolar spaces and the consequent creation of large areas of intrapulmonary shunt. However, two patients have undergone a single lung transplantation with acceptable results and have had no evidence of recurrence in the transplanted lung thus far. Furthermore, even for bilateral transplantation, it is not known whether PAM can recur. Recurrence after transplantation has not been reported to date, suggesting that, in fact, PAM is a result of local inflammatory responses or a genetically determined error in alveolar metabolism, rather than a systemic disorder.

lung transplantation has some drawbacks because not all patients are eligible for transplantation as factors such as age and comorbidities may pose an unacceptably high risk. In addition, patients with end-stage lung disease often become critically ill and may not survive until a donor organ is available. Lung transplantation creates new medical problems and the lifeexpectancy after this operation remains limited.

\section{Acknowledgment:-}

None

Source(s) of support in the form of grants, equipment, drugs

None

\section{Conflicts of Interests:-}

None

\section{Conclusion:-}

we successfully performed a double lung transplantation in a case of respiratory failure resulting from end-stage PAM. Although elderly recipients with end-stage PAM may have technically difficult problems related to surgery, such transplantation can be done safely and successfully when sufficient strategies are used.

\section{References:-}

1. Lauta VM. Pulmonary alveolar microlithiasis: an overview of clinical and pathological features together with possible therapies. Resp Med. 2003;97:1081-5.[PubMed]

2. Castellana G, Gentile M, Castellana R, Fiorente P, Lamorgese V. Pulmonary Alveolar Microlithiasis: Clinical Features, Evolution of the Phenotype, and Review of Literature. Am J Med Gen. 2002;111:220-4.[PubMed]

3. Castellana G, Lamorgese V. Pulmonary Alveolar Microlithiasis: World Cases and Review of Literature. Respiration. 2003;70:549-55.[PubMed] 